\title{
On the Behaviour of the Distributional Stieltjes Transformation at the Origin
}

\section{S. Primović}

Es wird das Verhalten der Stieltjes-Transformierten solcher Distributionen aus $\mathscr{S}_{+}^{\prime} \mathrm{im}$ Ur. sprung 0 untersucht, die ein geeignetes quasiasymptotisches Verhalten in $0^{+}$besitzen. Diese : leuen Ergebnisse werden mit Hilfe bekannter Résultate über das asymptotische Verhalten bei $\infty$ erhalten. Ferner wird auch ein Satz vom Tauberschen Typ ïber das Verhalten in 0 bewiesen.

Исследуется поведение трансформации Стильтьеса таких дистрибуций иа $\mathscr{S}_{+}^{\prime}$ в начале координат 0 , которые имеют подходящее квазиасимптотическое поведение в точке $0^{+}$. Эти новые результаты получаются с помощью известных об асимптотическом поведении в точке $\infty$. Доказыпается также один результат Тауберова типа о поведении в точке 0.

The behaviour of the distributional Stieltjes transformation at the origin 0 is investigated for distributions of $\mathscr{F}_{+}^{\prime}$ having appropriate quasiasymptotic behaviour at $0^{+}$. These new results follow by known ones for the asymptotic behaviour at $\infty$. A Tauberian-type result for the behaviour at 0 is also obtained.

\section{Notions and known results}

The sets of real and natural numbers are denoted by $\Re$ and $\Re$, respectively. $\mathscr{S}(\Re)=\mathscr{S}$ and $\mathscr{S}^{\prime}(\Re)=\mathscr{S}^{\prime}$ denote the spaces of rapidly decreasing functions and tempered distributions, respectivelý. The space $\mathcal{J}^{\prime}(r), r \in \mathfrak{R} \backslash(-\mathfrak{R})$ is defined in [3] as a subspace of $\mathscr{S}_{+}^{\prime}=\left\{f \in \mathscr{S}^{\prime}(\Re)\right.$; supp $\left.f \subset[0, \infty)\right\}$ consisting of all $f$ of the form.

$$
\begin{aligned}
& f=D^{m} F, \text { for some } m \in \mathfrak{N}_{0}=\mathfrak{N} \cup\{0\}, \\
& F \in L_{\mathrm{loc}}^{1}, \quad \operatorname{supp} F \subset[0, \infty),
\end{aligned}
$$

$D$ is the distributional derivative, such that

$$
\int_{0}^{\infty}\left|F^{i}(t) /(t+x)^{r+m+1}\right| d t<\infty, \quad ; x>0 .
$$

Obviously, if $f \in \mathcal{J}^{\prime}(r+p)$, then $f^{(p)} \in \mathcal{J}^{\prime}(r), p \in \mathfrak{R}_{0}$. We also need the definition of the space $\mathcal{J}^{\prime}(r), r \in \Re \backslash(-\mathfrak{R})$. This is a subspace of $\mathcal{J}^{\prime}(r)$ consisting of all $f \in \mathcal{J}^{\prime}(r)$ for which (1) holds and instead of (2), there holds

$$
|F(t)|<C(1+t)^{r+m-\varepsilon}, \quad t>0, \quad \text { for some } C, \varepsilon>0 \text {. }
$$

The distributional Stieltjes transformation of $f=D^{m} F^{\prime} \in \gamma^{\prime}(r)$ is defined by [2]

$$
\left(S_{r} f\right)(z)=(r+1)_{m} \int_{0}^{\infty} \frac{F(t) d t}{(z+t)^{r+m+1}}, \quad z \dot{\in} \mathfrak{C} \backslash(-\infty, 0],
$$


where $(a)_{m}=a(a+1) \ldots(a+m-1), m \in \mathfrak{R},(a)_{0}=1, a \in \mathfrak{R}$ and $\mathfrak{S}$ is the set of complex numbers. This is a holomorphic function.

We always denote in this paper by $L$ a function which is slowly varying at $\infty\left(0^{+}\right)$, i.e. which is a continuous positive function defined in $(0, \infty)$ such that $L(\lambda x) / L(x) \rightarrow 1$ as $x \rightarrow \infty\left(x \rightarrow 0^{+}\right), \lambda>0$. For the properties of such functions we refer to [7]. When in connection with a function $L$ we deal with the point $\infty\left(0^{+}\right)$we shall always assume that $L$ is slowly varying at $\infty\left(0^{+}\right)$.

In our investigations of the distributional Stieltjes transformation the notions of quasiasymptotic behaviour at $\infty$ and at $0^{+}$play a fundamental role. These notions are introduced by Zavialov [9]. Note that in [6] we changed slightly the definition of the quasiasymptotic behaviour at $0^{+}$. Recall, $f \in \mathscr{I}_{+}^{\prime}$ has the quasiasymptotic behaviour at $\infty\left(0^{+}\right)$related to $k^{\alpha} L(k)\left((1 / k)^{\alpha} L(1 / k)\right)$ with the limit $g \epsilon_{\mathcal{F}_{+}^{\prime}}$ if

$$
\begin{aligned}
& \lim _{k \rightarrow \infty}\left\langle\frac{f(k x)}{k^{\alpha} L(k)}, \varphi(x)\right\rangle=\langle g(x), \varphi(x)\rangle, \quad \varphi \in \mathscr{I}, \\
& \left(\lim _{k \rightarrow \infty}\left\langle\frac{f(x / k)}{(1 / k)^{\alpha} L(1 / k)}, \varphi(x)\right)=\langle g(x), \varphi(x)\rangle, \quad \varphi \in \mathscr{J}\right) .
\end{aligned}
$$

$\langle\cdot, \cdot\rangle$ is the dual pairing between $\mathscr{S}^{\prime}$ and $\mathscr{J}$. We include in the definition the case $g=0$, as well, while in [9] and [6] were assumed $g \neq 0$. It is well known that $g$ in (4) nust be of the form $g=C f_{a+1}$, where

$$
f_{a+1}(t)=\left\{\begin{array}{ll}
H(t) t^{\alpha} / \Gamma(\alpha+1) & \text { if } \alpha>-1, \\
D^{n} f_{\alpha+n+1}(t) & \text { if } \alpha \leqq-1
\end{array} \quad(t \in \mathfrak{R})\right.
$$

for some $n \in \mathfrak{R}$ with $n+\alpha>-1$ see [8]; $H$ is Heviside's function, $\Gamma$ is the gamma function. For the properties of the quasiasymptotic behaviour at $\infty$ we refer to [8] and at $0^{+}$we refer to [6]. Let us only quote the so-called structural theorem. Let $f \in \mathscr{I}_{+}^{\prime}$ have the quasiasymptotic behaviour at $\infty\left(0^{+}\right)$related to $k^{\alpha} L(k)\left((1 / k)^{\alpha} L(1 / k)\right)$ with the limit $C f_{a+1}$; then there is an integer $m_{0} \in \mathfrak{N}_{0}, m_{0}+\alpha>-1$, such that for every $m \geqq m_{0}$ there is a locally integrable function $F_{m}$ with supp $F_{m} \subset[0, \infty)$ such that

$f=F_{m}^{(m)}, \quad \lim _{x \rightarrow \infty} \frac{F_{m}(x)}{x^{\alpha+m}}=\frac{C}{\Gamma(\alpha+m+1)} \quad\left(\lim _{x \rightarrow 0^{+}} \frac{F_{m}(x)}{x^{\alpha+m}}=\frac{C}{\Gamma(\alpha+m+1)}\right)$.

Note that (5) is proved in [8] and [6] with the assumption $C \neq 0$. One can easily prove that this holds with $C=0$, as well.

We shall need the following theorem from [5].

Theorem A: Let $f \in \mathscr{S}^{\prime}$ have the quasiasymptotic behaviour at $\infty$ related to $k^{\alpha} L(k)$ with the limit $C f_{a+1}$, where $\alpha<r$. Then.

$$
\lim _{k \rightarrow \infty} \frac{(k s)^{r-\alpha}}{L(k)}\left(S_{r} f\right)(k s)=\frac{C \Gamma(r-\alpha)}{\Gamma(r+1)}
$$

for any $s \in \Omega_{\varepsilon}=\left\{\varrho \mathrm{e}^{\mathrm{i} \varphi}: \varrho>0,-\pi+\varepsilon \leqq \varphi \leqq \pi-\varepsilon\right\}$, where $0<\varepsilon<\pi / 2$. If $L=1$, then

$$
s^{r-\alpha}\left(S_{r} f\right)(s) \rightarrow \frac{C \Gamma(r-\alpha)}{\Gamma(r+1)} \quad \text { uniformly in } \Omega_{\varepsilon} \text { when } \quad|s| \rightarrow \infty
$$

This theorem was proved with the assumption $C \neq 0$. But it also holds with $C=0$, because the main step of its proof is the use of (5) which holds with $C=0$.

As well, we need from [4] the following theorem: 
The orem B: The following two statements are true: $\begin{aligned} & \text { (i) Let } \Phi \text { be integrable on } \Re, \operatorname{supp} \Phi \subset[\overline{0}, \infty) \text { and } C=\int_{0}^{\infty} \Phi(t) d t \text {. Then } \lim \Phi(k \cdot) / k^{-1} \\ = & C \delta \text { in } \mathscr{S}^{\prime}(\delta \text { is the delta distribution }) .\end{aligned}$

(ii) Let $\Phi \in L_{\mathrm{loc}}^{1}$, supp $\Phi \subset[0, \infty), \lim _{t \rightarrow \infty} \Phi(t) / t^{-1} L(t)=C$ and let $L^{*}(x)=\int_{a}^{x}(L(t) / t) d t$ $\rightarrow \infty$ as $x \rightarrow \infty(a>0)$. Then $\lim _{k \rightarrow \infty} \Phi(k \cdot) / k^{-1} L(k)=C \delta$ in. $\mathscr{S}^{\prime}$.

Note that (i) holds trivially and that (ii) follows from the fact that

$$
\int_{0}^{x} \Phi(t) d t / L^{*}(x) \rightarrow C, \quad x \rightarrow \infty \quad \text { (L'Hospital's rule) }
$$

and so

$$
\lim _{k \rightarrow \infty} \int_{0}^{k x} \Phi(t) d t / L^{*}(k)=C H(x) \text { in } \mathscr{S}^{\prime} \quad(x \in \Re)
$$

Now, by differentiation we obtain (ii).

Note that we include. in Theorem $B$ the case $C=0$.

Remark: By developing the notion of the quasiasymptutic at $0^{+}$we proved in [6] the same theorem for the behaviour of $S_{r} f$ at 0 : If $f \in \gamma^{\prime}(r)$ has the quasiasymptotic behaviour at $0^{+}$related to $(1 / k)^{\alpha} L(1 / k),{ }^{\alpha} \alpha<r$, then (6) holds with $1 / k$ instead of $k$ and (7) holds with $|s| \rightarrow 0$ instead of $|s| \rightarrow \infty$. The aim of this paper is to extend this theorem using Theorem $\mathrm{A}$. We shall also give a Tauberian-type result. It is based on the following theorem from [2: p. 339].

Theorem C: Let us suppose that for some $m>0$

$$
\int_{0}^{\infty} d \varphi(\lambda) /(\lambda+x)^{m+1} \sim \int_{0}^{\infty} d \psi(\lambda) /(\lambda+x)^{m+1}, \quad x \rightarrow \infty,
$$

and that the following conditions are satisfied:

1. $\varphi$ and $\psi$ are non-decreasing;

2. $\psi(x) \rightarrow \infty$ as $x \rightarrow \infty$;

3. for any $C>1$ there are $\gamma$ and $N, 0<\gamma<m, N>0$, such that for any $x>y>N$, $\psi(x) / \psi(y)<C(x / y)^{y}$.

Then, $\varphi(\hat{\lambda}) \sim \psi(\hat{\lambda}), \lambda \rightarrow \infty$.

\section{Abelian-type results}

Theorem 1: Let $F \in, J^{\prime}(r)$ have the quasiasymptotic behaviour al $0^{+}$related to $(1 / k)^{\circ}$ $\times L(1 / k)$ with the limit $C f_{a+1}$. Then:

$$
\text { For } r>\alpha, \lim _{x \rightarrow 0^{+}} \frac{\left(S_{r} f\right)(x)}{x^{\alpha-r} L(x)}=\frac{C \Gamma(r-\alpha)}{\Gamma(r+1)}
$$

(ii) For $r<\alpha, \lim _{x \rightarrow 0^{+}}\left(S_{r} f\right)(x)=B$, for some $B \in \Re$ : 
(iii) Assume that $r=\alpha$ and that $L_{1}(t)=L(1 / \bar{t}), t>\dot{0}$, is locally integrable in $(a, \infty)$, for some $a>0$. If

$$
\int_{a}^{\infty} \frac{L_{1}(t)}{t} d t\left\{\begin{array}{l}
<\infty, \text { then } \lim _{x \rightarrow 0^{+}}\left(S_{r} f\right)(x)=B, \\
=\infty, \text { then } \lim _{x \rightarrow 0^{+}}\left(\left(S_{\mathrm{f}} f\right)(x) / \tilde{L}(x)\right)=B
\end{array}\right.
$$

for some $B \dot{\epsilon} \mathfrak{R}$, where $\mathscr{L}(1 / x)=L_{1}(x)=\int_{a}^{x}\left(\left(L_{1}(t) / t\right)\right) d t, x>0$.

Note that $B$ in part (ii) and in both cases of part (iii) denotes always different constants ' which depends on $C, \alpha$ and $r$. This dependence will be clear from the proof. Clearly, (i) is a part of the assertion given in the Remark. We shall give here another proof of this fact.

Proof: Assume that (1) and (2)* hold for $f$ with some $\bar{m} \in \mathfrak{R}_{0}$. Then for $F_{m}$ defined by

$$
F_{m}(x)=\int_{0}^{x} F_{m-!}(l) d l \quad\left(x \in \mathfrak{R} ; m=\bar{m}+1, \bar{m}+2, \ldots, F_{\bar{m}}=F\right)
$$

there holds:

$$
\begin{aligned}
& F_{m} \text { is continuous, supp } F_{m} \subset[0, \infty) \\
& \left|F_{m}(t)\right| \leqq C_{m}(1+t)^{r+m-t}, \quad t>0, \quad F_{m}^{(m)}=t \\
& \quad \vdots \\
& \left(S_{r} f\right)(z)=(r+1)_{m} \int_{0}^{\infty} \frac{F_{m}(t)}{(z+t)^{r+m+1}} d t, \quad z \in[(-\infty, 0]
\end{aligned}
$$

(for $\varepsilon$ see $(2)^{*}$ ). Note that the functions $F_{m}$ are uniquely determined. The structural theorem at. $0^{+}$given in (5) implies that, for $m \geqq \max \left\{m_{0}, \bar{m}\right\}, \lim _{x \rightarrow 0^{+}}\left(F_{m}(x) / x^{\alpha+m}\right)$ $=C / \Gamma(\alpha+m+1)$. Fix $m>\max \left\{m_{0}, \bar{m}\right\}$ and denote $F_{n}$ by $F$ again. We have, for $k>0$ and $z \in \subseteq(-\infty, 0]$,

$$
\begin{aligned}
& \left(\frac{z}{k}\right)^{r+m+1}\left(S_{f}\right)\left(\frac{z}{k}\right)=(r+1)_{m}\left(\frac{z}{k}\right)^{r+m+1} \cdot \int_{0}^{\infty} \frac{F(t) d t}{(t+z / k)^{r+m+1}} \\
& =(r+1)_{m}\left(\frac{z}{k}\right)^{r+m+1} \int_{0}^{\infty} \frac{(1 / u)^{2} F(1 / u) d u}{(1 / u+z / u)^{r+m+1}}=(r+1)_{m} \int_{0}^{\infty} \frac{u^{r+m-1} F(1 / u) d u}{(u+k / z)^{r+m+1}} .
\end{aligned}
$$

So, we obtain, for $k>0$ and $z \in \dot{\mathfrak{C}} \backslash(-\infty, 0]$,

$$
\left(\frac{z}{k}\right)^{r+m+1}\left(\dot{S}_{r} f\right)\left(\frac{z}{k}\right)=(r+1)_{m}\left(S_{r+m} \Phi\right)\left(\frac{k}{z}\right)
$$

where $\Phi(t)=t^{r+m-1} F(1 / t)$ for $t>0$ and $\Phi(t)=0$ for $t \leqq 0$. Obviously,

$$
\lim _{t \rightarrow \infty}\left(\Phi(t) / t^{r-\alpha-1} L_{1}(t)\right)=C / \Gamma(\alpha+m+1)
$$

$\left(L_{1}^{\prime}(k)=L(1 / k)\right.$ is slowly varying at $\left.\infty\right)$. Because of $(8)$ we have (with suitable $C_{1}$ )

$$
|\Phi(t)| \leqq C_{1} t^{-1+\cdot \varepsilon}(1+t)^{r+m-\varepsilon}, \quad t>0 .
$$

((11) shows that $\Phi$ is locally integrable on $\Re$.) 
(i) Assume $\alpha<r$. Since $r-\alpha-1>-1$, (10) implies that $\Phi$ has the quasiasymptotic behaviour at $\infty$ related to $k^{r-\alpha-1} L_{1}(k)$ with the limit $C(\Gamma(r-\alpha) / \Gamma(\alpha+m$ +1 )) $f_{r-a}[8]$. So, Theorem A implies (i).

(ii) Assume $\alpha>r$. Now, from (10) (and (11)) it follows that $\Phi$ is integrable on $\Re$. From T'heorem $B /(i)$ it follows that $\Phi$ has the quasiasymptotic behaviour at $\infty$ related to $k^{-1}$ with the limit $\widetilde{B} \delta$ where $\widetilde{B}$ depends on $m$ and $\Phi$ (see Theorem B). Theorem implies (ii): We have by $(9)\left(S_{r} f\right)(1 / k)=k^{r+m+1}(r+1)_{m}\left(S_{r+m} \Phi\right)(k) \rightarrow(r+1)_{m} \tilde{B}$, $k \rightarrow \infty$, i.e. $\left(S_{r} f\right)(t) \rightarrow B=(r+1)_{m} \tilde{B}, t \rightarrow 0^{+}$. Note that $B$ does not depend on $m$.

(iii) Assume $\alpha=r$. We have by $(10), \Phi(t) \sim \vec{B} t^{-1} L_{1}(t), t \rightarrow \infty$, where $\vec{B}$ is a suitable constant. If $\int_{a}^{\infty}\left(\left(L_{1}(t) / t\right)\right) d t<\infty$, then from Theorem $B /(\mathrm{i})$ it follows that $\Phi$ has the quasiasymptotic behaviour at $\infty$ related to $k^{-1}$ with the limit $\tilde{B} \delta$ where $\tilde{B}$ clepends on $m$ and $\Phi$. Theorem A completes the proof of the first part of (iii). Assume now that $\alpha=r$ and $\int_{a}^{\infty}\left(\left(L_{1}(t) / t\right)\right) d t=\infty$. Then (10), Theorem B/(ii) and Theorem 'A completes the proof of (iii), because $\Phi$ has the quasiasymptotic behaviour at $\infty$ with the limit $\tilde{B} \delta$ related to $k^{-1} \tilde{L}(k)$

Let us set for $\varepsilon, 0<\varepsilon<\pi / 2$,

$$
L(0, R)=\{s:|s|<R\}, \quad \Lambda_{i}=\left\{\varrho \mathrm{e}^{\mathrm{i} \varphi}: \varrho>0,|\varphi| \leqq \pi-\varepsilon\right\} .
$$

Lemma 2: Let $f$ satisfy the conditions of Theorem 1 with $\alpha \geqq r>-1$. Then the functions

$$
\begin{aligned}
& s \rightarrow\left(S_{r} f\right)^{-}(s), \quad s \in \Lambda_{\varepsilon} \cap L(0, R), \quad \text { for } \alpha>r, \\
& s \rightarrow \frac{1}{\ln s}\left(S_{r} f\right)(s), \quad s \in \Lambda_{c} \cap L(0, R), \quad \text { for } \alpha=r, L=1,
\end{aligned}
$$

are bounded (ln $s=\ln |s|+\mathrm{i} \varphi,|\varphi| \leqq \pi-\varepsilon)$.

Proof: Observe first the case $\alpha>r$. Clearly it is enough to prove that $S_{r} f$ is bounded in $\Lambda_{\varepsilon} \cap L(0, R)$. For $z=\varrho \mathrm{e}^{\mathrm{i} \varphi} \in \Lambda_{\varepsilon} \cap L(0, R)$ we have $|1 / z| /|t+1 / z|$ $=\lambda\left(t^{2}-2 t \lambda \cos \varepsilon+\lambda^{2}\right)^{-1 / 2}, \lambda=1 / \varrho,>1 / R$. From

$$
\begin{aligned}
t^{2}-2 t \lambda \cos \varepsilon+\lambda^{2} & \geqq t^{2}+\lambda^{2}+\left(t^{2}+\lambda^{2}\right) \cos \varepsilon-(t+\lambda)^{2} \cos \varepsilon \\
& \geqq\left(t^{2}+\lambda^{2}\right)(1+\cos \varepsilon)-2\left(t^{2}+\lambda^{2}\right) \cos \varepsilon \\
& =\left(t^{2}+\lambda^{2}\right)(1-\cos \varepsilon)>(t+\lambda)^{2}(1-\cos \varepsilon) / 2
\end{aligned}
$$

we have $(\lambda=|1 / z|)$

$$
\left|\frac{1 / z}{t+1 / z}\right|<\left(\frac{2}{1-\cos \varepsilon}\right)^{1 / 2} \frac{\lambda}{t+\lambda}, \quad t>0, z \in \Lambda_{\varepsilon} \cap L(0, R) .
$$

This implies that, for suitable $C,|1 / z| /|t+1 / z| \leqq C\left(t>0, z \in A_{\varepsilon} \cap L(0, R)\right)$. Since (9) implies

$$
(S, f)(z)=\frac{(r+1)_{m}}{z^{r+m+1}} \int_{0}^{\infty} \frac{\Phi(t) d t}{(t+1 / z)^{r+m+1}}, \quad z \in \mathbb{E} \backslash(-\infty, 0]
$$

and $\Phi$ is integrable, we have

$$
\left|\left(S_{f} f\right)(z)\right| \leqq C^{r+m+1} \int_{0}^{\infty}|\Phi(t)| d t<\infty, \quad z \in \Lambda_{e} \cap L(0, R)
$$


Observe now the case $\alpha=r, L=1$. In this case in Theorem $1 /(\mathrm{iii})$, second case, we have $\tilde{L}(x) \sim-\ln x, x \rightarrow 0^{+}$. Since the limit $\lim _{x \rightarrow \infty} \Phi(x) / x^{-1}$ is finite, with suitable $A, B \in \Re$, and $s \in A_{\varepsilon} \cap L(0, R), \lambda=1 /|s|,(9),(11)$ and (12) imply

$$
\begin{aligned}
\left(S_{r} f\right)(s) & \leqq(r+1)_{m}\left(\int_{0}^{A}|\Phi(t)|\left|\frac{1 / s}{t+1 / s}\right|^{r+m+1} d t+B \int_{A}^{\infty} \frac{1}{t}\left|\frac{1 / s}{t+1 / s}\right|^{r+m+1} d t\right) \\
& \leqq(r+1)_{m}\left(\int_{0}^{A}|\Phi(t)| d t+B\left(\frac{2^{2}}{1-\cos \varepsilon}\right)^{r+m+1} \int_{A}^{\infty} \frac{\lambda^{r+m+1} d t}{t(\lambda+t)^{r+m+1}}\right) .
\end{aligned}
$$

From the identity

$$
\frac{1}{t}\left(\frac{\lambda}{\lambda+t}\right)^{r+m+1}=\frac{1}{t}-\frac{1}{t+\lambda}-\frac{\lambda}{(t+\lambda)^{2}}-\cdots-\frac{\lambda^{r+m}}{(t+\lambda)^{r+m+1}}
$$

we have

$$
\int_{\lambda}^{\infty} \frac{1}{t}\left(\frac{\lambda}{\lambda+l}\right)^{r+m+1} d t=\left.\left(\ln \frac{t}{t+\lambda}+\frac{\lambda}{t+\lambda} \cdots+\frac{1}{(r+m)} \frac{\lambda^{r+m}}{(t+\lambda)^{r+m}}\right)\right|_{A} ^{\infty}
$$

We obtained that the integral $\int_{A}^{\infty} \ldots$ is bounded independently of $\lambda$. This implies that $\left(S_{r} f\right)(s), s \in \Lambda_{\varepsilon} \cap L(0, R)$, is bounded. Sirice $1 / \ln s, s \in A_{\varepsilon} \cap L(0, R)$, is -bounded, as well, the proof is complete

Assume that the conditions of Iemma 2 hold. We set

$$
A(z)=\left\{\begin{array}{ll}
\left(S_{r} f\right)\left(\frac{1}{z}\right) & \text { if } \alpha>r, \\
\frac{1}{\ln z}\left(S_{r} f\right)\left(\frac{1}{z}\right) & \text { if } \alpha=r, L=1
\end{array} \quad\left(z \in A_{\varepsilon}+\frac{1}{R}\right)\right.
$$

Lemma 2 implies that in both case $A$ is bounded in $A_{z}+1 / R$. Set $A_{1}(z)=A(z+1 / R)$, $z \in \Lambda_{\varepsilon}$.

Lemma 3: There holds $A_{1}(z) \rightarrow B$ uniformly in $\Lambda_{\varepsilon}$ when $|z| \rightarrow \infty$, where $B$ is from Theorem $1 /$ (ii) or (iii), second case.

Proof: We have that $A_{1}$ is bounded in $\Lambda_{\varepsilon}$ and that $A_{1}(x) \rightarrow B, x \rightarrow \infty$ (Theorem 1 ) (ii) or (iii)). So Montel's theorem [1: p. .5] implies the assertion

The orem 4: Assume that the conditions of Theorem 1 hold for $f$ with $\alpha \geqq r>-1$.

(i) If $\alpha>r$, then $\left(S_{r} f\right)(z) \rightarrow B,|z| \rightarrow 0, z \in \Lambda_{\varepsilon}$, uniformly.

(ii) If $\alpha=r, L=1$, then $(1 / \ln z)\left(S_{f} f\right)(z) \rightarrow B,|z| \rightarrow 0, z \in \Lambda_{\varepsilon}$, uniformly.

Proof: Lemma 3 implies that in both cases $A(z) \rightarrow B,|z| \rightarrow \infty, z \in \Lambda_{\varepsilon}+1 / R$, uniformly. So, this implies the proof of the theorem 


\section{A Tauberian-type result}

Let $r \in \Re \backslash \Re$. Assume that $f=F^{(m)} \in \mathscr{S}_{+}^{\prime}$ where $F$ is a non-increasing positive locally integrable function such that $F(x)<A x^{r+m-\varepsilon}, x>0$, for some $A>0$. Assume that $s>1, r+m-s>0$ and that $x^{r+m-s} L_{1}(x), x>A$, is non-decreasing, where $L(x), x>0$, is slowly varying at $0^{+}$and $L_{1}(x)=L(1 / x), x>0$. With the given assumptions we have

Theorem 5: Assume that

Then

$$
\left(S_{r} f\right)(x) \sim(r+1)_{m} \frac{\Gamma(s)}{\Gamma(r+m+1)} \frac{L(x)}{x^{m+r+1-s}}, \quad x \rightarrow 0+.
$$

$$
\lim _{k \rightarrow \infty} \frac{f(x / k)}{(1 / k)^{s-m-1} L(1 / k)}=B f_{s-m} \text { in } \mathscr{S}^{\prime},
$$

where $B$ is a suitable constant.

Proof: The assumption of the theorem and (9) imply that

$$
\left(S_{r+m} \Phi\right)(x) \sim \frac{\Gamma(s)}{\Gamma(r+m+1)} \frac{L_{1}(x)}{x^{s}}, \ldots x \rightarrow \infty,
$$

where $\Phi(x)=x^{r+m-1} F(1 / x), x>0$, is a non-decreasing function. Let

$$
\psi(x)= \begin{cases}x^{r+m-8} L_{1}(x) / \Gamma(r+m-s+1), & x>A \\ 0, & x \leqq A\end{cases}
$$

Theorem A implies

So,

$$
\int_{0}^{\infty} \frac{d \psi(t)}{(x+t)^{r+m}}=(r+m) \int_{0}^{\infty} \frac{\psi(t)}{(x+t)^{r+m+1}} d t \underset{x \rightarrow \infty}{\sim} \frac{(r+m) \Gamma(s)}{\Gamma(r+m+1)} \frac{L_{1}(x)}{x^{s}}
$$

$$
\int_{0}^{\infty}\left(d \Phi /(x+t)^{r+m}\right) \sim \int_{0}^{\infty}\left(\left(d \psi /(x+t)^{r+m}\right)\right) \text { as } x \rightarrow \infty .
$$

If we show that for every $C>1$ there are constants $\gamma$ and $N, 0<\gamma<r+m-1$, $N>0$, such that

$$
x>y>N \Rightarrow x^{r+m-s} L_{1}(x) / y^{r+m-\diamond} L_{1}(y)=C(x / y)^{r},
$$

then all the assumptions of Theorem $B$ are satisfied and this theorem implies

$$
\Phi(x) \sim \psi(x), \quad \cdots x \rightarrow \infty
$$

Take $\gamma=r+m-s+\varepsilon$ where $\varepsilon>0$ such that $\gamma>0$ and $\varepsilon<s-1$. With such $\gamma$ and $x=\lambda y, \lambda>1, y>N,(14)$ becomes $L_{1}(\lambda y) \leqq C \lambda^{e} L_{1}(y)$; and this is true [7:p. 18]; note, $N$ depends on $C$. So, (15) implies $\varphi(x) \sim x^{r+m-s} L_{1}(x) / \Gamma(r+m-s+1), x \rightarrow \infty$, and thus

i.e.

$$
x^{r+m-1} F\left(\frac{1}{x}\right) \sim \frac{x^{r+m-s}}{\Gamma(r+m-s+1)} L_{1}(x), \quad x \rightarrow \infty
$$

$$
F(x) \sim \frac{x^{-1}}{\Gamma\left(r+m-s^{+}+1\right)} L(x), \quad x \rightarrow 0^{+} .
$$

Since $f=F^{(m)}$ we have for suitable $B$ the assertion 
Acknowledgement. This material is based on work supported by the U.S. - Yugoslav Joint Fund for Scientific and Technological Cooperation, in cooperation with the (IFP) under Grant 838.

\section{REFERENCES}

[1] Boas, R. P.: Entire Functions. New York: Acad. Press 1954.

[2] Владимиров, В. С., Дрожжинов, Ю. Н., и Б.И. ЗАвьялов: Многомерные Таубберовы теоремы для обобщенных функций. Москва: Изд-во Наука 1986.

[3] ЗАВВ̈лов, Б. И Автомодельная асимптотика әлектромагнитных форм-факторов и поведение их фурье-обраӟов в окрестности светового копуса. Теор. Мат. Физ. 17 (1973), $178-188$.

[4] Костючғико; А. Г., и И! С. Саргсян : Распределение собственных значений. Москва: Изд-во Наука 1979.

[5] Lavoine, J., and D. P. Misra: Abelian theorems for the distributional Stieltjes transformation. Proc. Camb. Phil. Soc. 86 (1979), 287-293.

[6] Pilmović, S., and A. TAKÁ̌r : The quasiasy mptotic behaviour of some distributions. Rev. Res. Fac. Sci. Univ. Novi Sad 15 (1985), 37-46.

[7] Primović, S., and B. Stanković: Abelian Theorem for the Distributional-Stieltjes Trans. form. Z. Anal. Anw. 6 (1987), $341-349$.

[8] Puripović, S., and B. Stanković: Initial value Abelian theorems for the distributional Stiletjes transform. Studia Math. (to appear).

[9] Seneta, E.: Regularly Varying Functions (Lect. Notes Math. 508). Berlin-HeidelbergNew York: Springer-Verlag 1976.

Manuskripteingang: 02.06. 1987; in revidierter Fassung 09.12.1987

\section{VERFASSER:}

Prof. Dr. Stevan Pilipović

Institute of Mathematics, University of Novi Sad

dr. Ilije Djuricića 4

Yu-21000 Novi Sad 